\title{
The late-time expansion of the ejecta of SN 1987A*
}

\begin{abstract}
R. A. Jansen and P. Jakobsen
Astrophysics Division, Space Science Department of ESA, ESTEC, NL-2200 AG Noordwijk, The Netherlands e-mail: rjansen@astro.estec.esa.nl; pjakobse@astro.estec.esa.nl

Received 11 January 2001 / Accepted 19 February 2001

Abstract. The evolution of the shape and size of the ejecta of SN 1987A is analyzed over a period of $\sim 8$ years based on HST images and spectra taken between 1278 and 4336 days after the supernova outburst. We combine both proprietary and archival HST data obtained with the FOC, WFPC2 and STIS. The low resolution nearUV prism FOC spectrum obtained at day 3043 has not been described previously. Although the FWHM of the ejecta grew linearly over the time span studied, the appearance of the SN envelope also changed markedly with wavelength. At visible wavelengths $(\lambda \simeq 5000 \AA)$ the ejecta became progressively more elongated, reaching an ellipticity of $\epsilon \simeq 0.25$ by day 4000 . In the near-UV $(\lambda \simeq 2500 \AA)$, the ejecta remained closely circular $(\epsilon \leq 0.1)$ and $\sim 50 \%$ larger in angular extent than in the visible. The FOC prism observations show that the large extent of the SN envelope is confined to a grouping of resonance lines spanning Mg I $\lambda 2852, \operatorname{Mg}$ II $\lambda \lambda 2795,2802 \AA$ and several Fe II multiplets - thereby confirming that the larger size of the debris in the near-UV is due to scattering in these optically thick transitions compared to the optically thin forbidden and semi-forbidden transitions that dominate the visible spectrum. The available data are not of sufficient quality to detect the slight deviation from linear expansion expected for the outermost regions of the near-UV images as predicted by Chugai et al. (1997).
\end{abstract}

Key words. supernovae: individual (SN 1987A) — supernova remnants

\section{Introduction}

The remnant of SN 1987A and its surroundings have been extensively monitored with the Hubble Space Telescope $(H S T)$ since its commissioning, first with the aberated telescope, later with WFPC2 and the COSTAR-corrected FOC, and most recently using STIS. While much attention has been devoted to the study of the inner circumstellar ring (e.g., Panagia et al. 1991; Luo et al. 1994; Plait et al. 1995; Crotts et al. 1995) and its interaction with the SN blast wave (e.g., Sonneborn et al. 1998; Crotts \& Heathcote 2000; Michael et al. 2000), the evolution of the spatially resolved SN ejecta itself is also of considerable interest in its own right.

Jakobsen et al. (1991) demonstrated that the expanding envelope of SN 1987A was spatially resolved by HST already in the first FOC images taken 1278 days after the explosion. These data combined with subsequent FOC observations made on days 1754,2511 and 2533 showed that

Send offprint requests to: R. A. Jansen,

e-mail: rjansen@astro.estec.esa.nl

* Based on observations made with the NASA/ESA Hubble Space Telescope, obtained at the Space Telescope Science Institute (STScI) and from the data archive at the STScI, which is operated by AURA, Inc., under NASA contract NAS 5-26555. the outer envelope of SN 1987A was expanding linearly with time in a self-similar fashion (Jakobsen et al. 1994).

The early FOC observations also revealed that the size of the SN debris varies significantly with wavelength, appearing twice as large in the near-UV (F275W filter) compared to the visible (F501N [O III] filter). Jakobsen et al. (1993) suggested that this difference in apparent size is likely due to an opacity effect, with optical depth unity in the near-UV being reached further out in the expanding ejecta compared to the optically thin [O III] line which probes deeper into the expanding envelope. This interpretation was further refined based upon FOS observations obtained by Wang et al. (1996) and Chugai et al. (1997), who showed that the near-UV $(\lambda \lambda 2350-2900)$ spectrum of SN 1987A spanned by the FOC F275W filter is dominated by a dense grouping of resonance lines containing Mg I $\lambda 2852$ and Mg II $\lambda \lambda 2795,2802$ and several multiplets of Fe II. These authors also showed that the velocitywidths of the $\mathrm{Mg}$ I $\lambda 2852$ and $\mathrm{Mg}$ II $\lambda \lambda 2795,2802$ lines are in good agreement with the expansion velocities inferred from the FOC near-UV images, and considerably broader than those of the forbidden and semi-forbidden lines which dominate the visible spectrum.

In this paper we present previously unpublished FOC objective prism observations of SN 1987A taken on day 3043 which, by bringing together both imaging and 
spectroscopic information serve to further explore the cause of the wavelength dependence of the apparent size of SN 1987A. Our main finding is that the largest extent of the ejecta is indeed confined to the above grouping of high opacity near-UV resonance lines, thereby confirming this explanation for the change in apparent size with wavelength. We also compare these new FOC angular diameter measurements to matching measurements derived from archival WFPC-2 and STIS data spanning up to day 4336. We show that, provided care is taken to put the different measures onto a common system by taking the non-zero ellipticity of the SN 1987A envelope into account, the data show good agreement and are consistent with linear expansion of the SN 1987A envelope over the entire $\sim 8$ year time span probed by the publicly available HST observations.

At the time of writing, some 14 years after the explosion, the afterglow of SN 1987A proper has faded beyond the reach of $H S T$ - and is likely to remain so even when the Advanced Camera for Surveys is installed. This paper is therefore an attempt at summarizing the available $H S T$ data on the shape and expansion of the SN 1987 debris.

\section{Data}

\subsection{Day 3043 FOC observations}

Low resolution spectra of the remnant of SN 1987A were obtained using the COSTAR-corrected FOC on board HST on 1995 June 24 (JD 2,449,892; day 3043). The FOC was used in filterless mode with the F/96 near-UV objective prism inserted. A total exposure of $16683 \mathrm{~s}$ was obtained, divided over 12 exposures of $1221.5-1432.5 \mathrm{~s}$ each, with pointings dithered in a $0^{\prime \prime} .2$ pattern for optimal removal of reseau marks and flatfield features not taken out by the standard "pipeline" procedures.

With the $512 \times 1024$ pixel detector format used, non-linearity in extended objects sets in at a count rate of 0.08 counts $\mathrm{s}^{-1} \mathrm{pix}^{-1}$ and saturation at 0.37 counts s ${ }^{-1} \mathrm{pix}^{-1}$. The count rates in the spectrum of the SN remnant are within the linear regime even at $\mathrm{H} \alpha$ and [O III], but the optical portions of companion stars 2 and 3 (in the enumeration of West et al. 1987) are severely saturated due to the low dispersion of the objective prism at the longer wavelengths. The $H S T$ roll-angle (V3 position angle) during these observations was 15.2 degrees, causing the spectrum of companion star 2 to overlap the circumstellar ring at the position of the faint foreground star (Plait et al. 1992).

The individual exposures were reduced using the standard HST postprocessing software and subsequently combined, weighted by exposure time. To reduce the contamination of the SN remnant spectrum by the near-UV light of star 2, located 2".9 NW of SN 1987A, we used a matched (in wavelength) portion of the spectrum of star 3 , located $1^{\prime \prime} 6$ to the SE, as a PSF template. None of the stars in the field of view for which we observe the optical portion of the spectrum aproximates the brightness of star 3. For

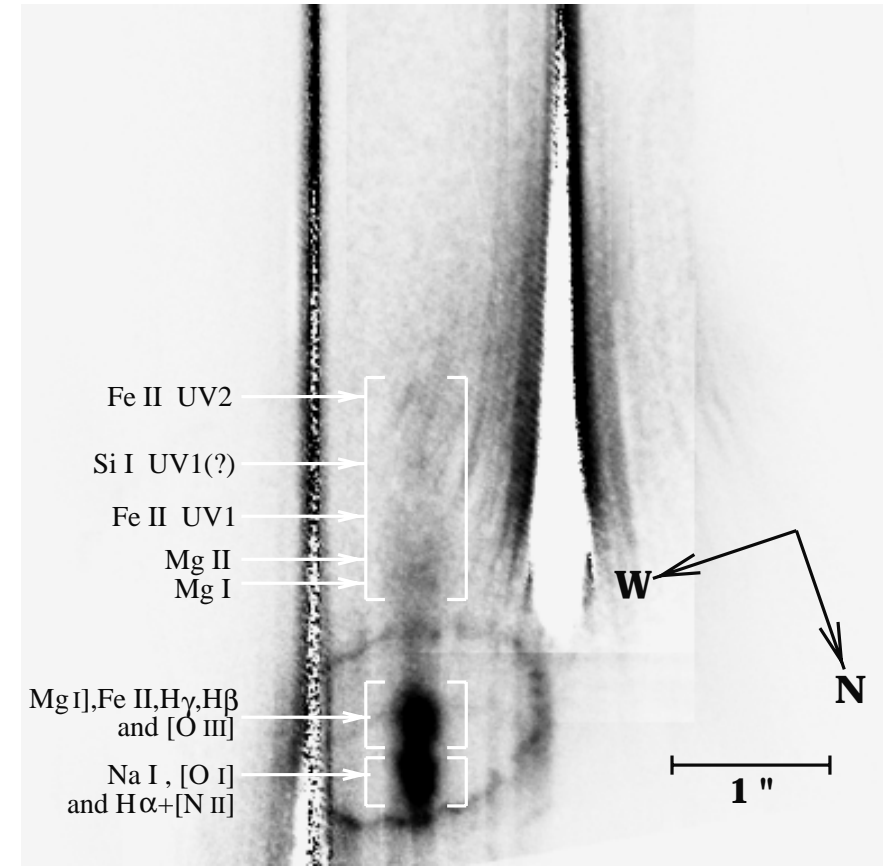

Fig. 1. Greyscale rendition of the portion of the FOC objective prism image that includes the dispersed image of the remnant of SN 1987A and the circumstellar ring in [O III] and (much fainter) in $\mathrm{H} \alpha+[\mathrm{N}$ II]. The spatial image scale and the image orientation are indicated. The spectra from companion stars 2 and 3 (located 2.' 9 NW and 1.' 6 SE of the SN remnant, respectively) have been partially removed. The $\lambda \lambda 2350$ $2900 \AA$ portion of the SN spectrum spanned by the grouping of $\mathrm{Mg}$ I/Mg II/Fe II near-UV resonance lines (see text) is indicated

subtraction of star 3 we therefore had to use a fainter star, located 7".9 S of SN 1987A as a template. Although the wings of the optical PSF in the template are not sampled to sufficiently faint levels to provide a good match, subtraction of this scaled-up template does significantly reduce the contamination from star 3 at the position of the SN remnant spectrum. In the following analysis, residual light from star 3 is the dominant source of error in the SN remnant spectrum for wavelengths shortward of $3000 \AA$.

The portion of the co-added and partially subtracted FOC image showing the (slitless) near-UV prism spectrum of SN 1987A is shown in Fig. 1. In this exposure, the circumstellar ring appears bright in the light of [O III] and is faintly discernable in $\mathrm{H} \alpha+[\mathrm{N}$ II] . The optical portion of the SN spectrum shows clear features, roughly located at $[\mathrm{O}$ III] and $\mathrm{H} \alpha+[\mathrm{N} \mathrm{II}]$, but resulting from blends of the lines of $\mathrm{Mg} \mathrm{I}], \mathrm{Fe}$ II, $\mathrm{H} \gamma, \mathrm{H} \beta$, and [O III] in the former and $\mathrm{NaI}$, $[\mathrm{O}$ I $]$ and $\mathrm{H} \alpha+[\mathrm{N} \mathrm{II}]$ in the latter case. The SN spectrum is traceable down to a wavelength of $\sim \lambda 2200 \AA$ in the nearUV. Moreover, consistent with the previous FOC imaging observations, the near-UV spectrum is noticeably more spatially extended than the visible spectrum.

In order to explore this quantitatively we selected two pixel regions centered on the $\mathrm{SN}$ remnant spectrum in the spatial direction and on wavelengths $2625 \AA$ and $4850 \AA$ in the dispersion direction. Along the dispersion 


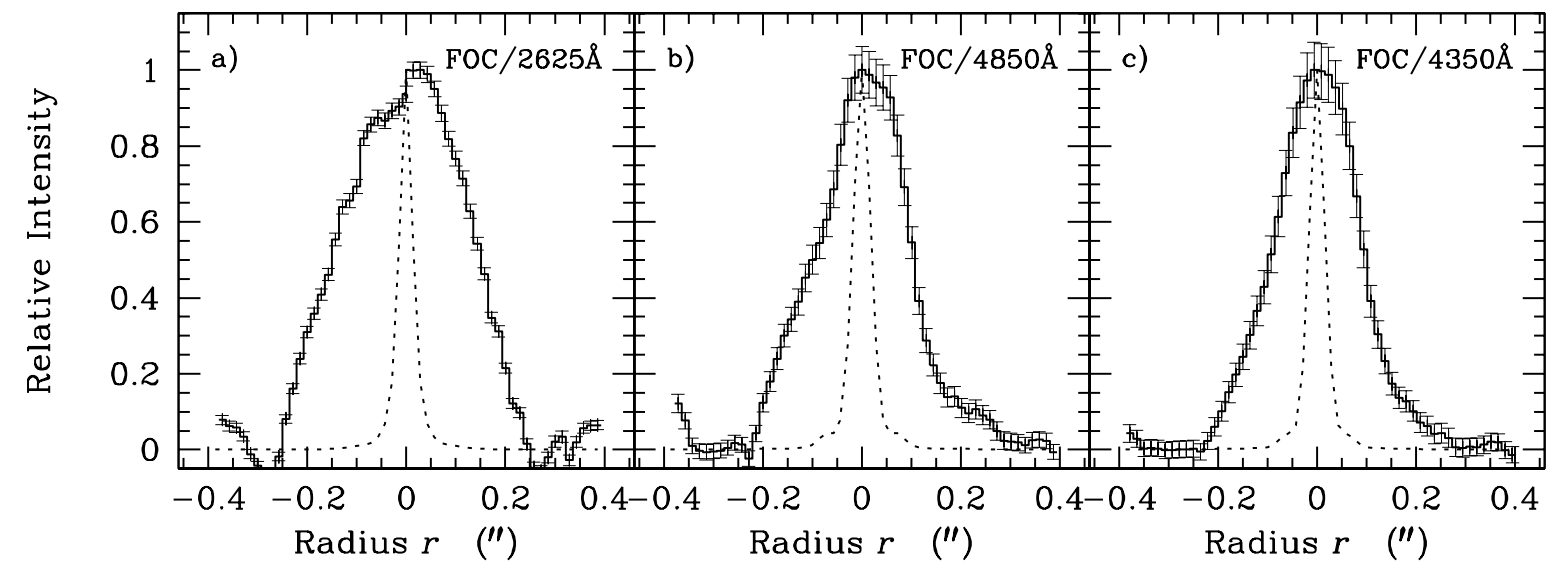

Fig. 2. Radial intensity profiles of the ejecta of SN 1987A in the day 3043 FOC data for a) the near-UV measured in a $550 \AA$ wavelength interval centered on $2625 \AA$ (comparable to the earlier F275W data and to more recent F255W filter WFPC2 data), and b) the optical in a $1300 \AA$ interval centered on $4850 \AA$ (comparable to the earlier F501N data [see text] and more recent F555W filter WFPC2 data), and c) a $700 \AA$ interval centered on $4350 \AA$ (matched to the WFPC2 F439W filter). The intensity profiles were normalized to the peak intensity. In each figure, the FOC PSF for the central wavelength of the interval is overlayed for comparison (dotted)

direction we averaged the pixel values in 93 lines and 26 lines, corresponding to $550 \AA$ and $1300 \AA$ wavelength intervals, respectively. This choice was a compromise between encompassing the respective $\mathrm{MgII} / \mathrm{Mg}$ I/Fe II and $\mathrm{Mg} \mathrm{I}] / \mathrm{H} \beta / \mathrm{Fe}$ II/[O III $]$ features (see below), and gathering enough signal to reduce the noise while avoiding contamination from the underlying image of the ring. The resulting radial intensity profiles are presented in Figs. 2a and $2 \mathrm{~b}$. The FOC PSF at $2625 \AA$ and $4850 \AA$ is overlayed in each figure. The apparent size of the SN debris as measured by the FWHM of the radial intensity profiles is $301 \pm 7$ mas at $2625 \AA$ and $201 \pm 10$ mas at $4850 \AA$. These values are corrected for the PSF. We also selected a third, 20 pixel $(700 \AA)$ wide region centered at $4350 \AA$, chosen to match a grouping of Fe II lines and the semi-forbidden Mg I] line (see Sect. 2.2.1). By sampling the same species ( $\mathrm{Mg}$ and $\mathrm{Fe}$ ), a comparison between the NUV and $4350 \AA$ line groupings is likely to be cleaner than a comparison with the $4850 \AA$ band, or even with the [O III] line. The apparent size (FWHM) of the debris measured in the radial intensity profile at $4350 \AA$ (Fig. 2c) is $188 \pm 13$ mas, smallest of any wavelength sampled in these data.

\subsection{Archival HST data}

The data on SN 1987A contained in the public HST archive consists of a large number of multifilter WFPC2 images, as well as STIS and FOS near-UV and optical spectra, and the earlier FOC imaging observations. The available data are summarized in Table 1. References to published observations are given in the final column of the table. A large portion of these data were obtained as part of the Supernova INtensive Study (SINS) project. Of the WFPC2 data, we only use the Planetary Camera chip (in the following referred to as $\mathrm{PC} 2$ ) which contains the image of the SN remnant itself.

\subsubsection{Spectroscopic observations and line identification}

In Fig. 3 we reproduce the 1995 January 7 (Day 2875) FOS spectrum presented in Chugai et al. (1997). The bandpasses of selected $H S T$ filters are overlayed to show which spectral features contribute most to the total signal in imaging observations through these filters. The identifications of the emission features were taken from Wang et al. (1996) and Chugai et al. (1997).

In the day 3880 STIS observations (1997, October 8) the $2250-3000 \AA$ wavelength region is very similar in appearance to the corresponding portion of the 1995 January 7 FOS spectrum of Fig. 3. The NUV features seen in these higher resolution spectra can also be gleaned in the dispersed 1995 June 24 FOC near-UV prism image (Fig. 1).

The 2350-2900 $\AA$ interval used to extract the radial intensity profile of Fig. 2a selects the strong NUV resonance lines of MgII, MgI and Fe II. The F255W (PC2) and F275W (FOC) filters are a good match to this ensemble of lines, although the latter places slightly more weight on the $\mathrm{Mg}$ lines. The narrow band filters F501N (FOC) and F502N (PC2) sample emission from [O III] and FeII $\lambda 5018 \AA$. The F555W (PC2) filter samples a multitude of lines: $\mathrm{Mg} \mathrm{I}], \mathrm{H} \beta$, [O III], Fe II, Na I, [O I], and $\mathrm{H} \alpha+[\mathrm{NII}]$ in its red tail. Nonetheless, comparisons of F501N and F502N filter data with data in this filter are still meaningful, since the dominant lines spanned by the F555W filter are all low opacity forbidden and semiforbidden transitions.

In the following we will treat the FOC F275W and PC2 F255W data as equivalent and sampling the resonance line dominated near-UV emission of SN 1987A. The FOC F501N and PC2 F502N, F555W and F547M imaging data are grouped in a similar manner as sampling the forbidden and semi-forbidden visible line emission from SN 1987A. 
Table 1. Overview of HST Data on SN 1987A used in this paper

\begin{tabular}{|c|c|c|c|c|c|c|c|c|}
\hline Day $^{\dagger}$ & Date & Instrument & Data type & Filter & Exposure $^{\ddagger}$ & Proposal $^{*}$ & P.I. & Reference \\
\hline \multirow[t]{2}{*}{1278} & Aug. 231990 & FOC & Imaging & F275W & 838 & 2999 & F. Macchetto & Jakobsen et al. (1991) \\
\hline & & & & F501N & 1660 & & & \\
\hline \multirow[t]{2}{*}{1754} & Dec. 131991 & FOC & Imaging & F275W & 2200 & 3874 & F. Macchetto & Jakobsen et al. (1993) \\
\hline & & & & $\mathrm{F} 501 \mathrm{~N}$ & 2200 & & & \\
\hline \multirow[t]{2}{*}{2511} & Jan. 081994 & FOC & Imaging & $\mathrm{F} 275 \mathrm{~W}+\mathrm{ND} 1$ & 4784 & 5186 & W. Sparks & Jakobsen et al. (1994) \\
\hline & & & & F $501 \mathrm{~N}$ & 4784 & & & \\
\hline \multirow[t]{2}{*}{2533} & Jan. 301994 & FOC & Imaging & F275W & 3586 & 5152 & P. Jakobsen & Jakobsen et al. (1994) \\
\hline & & & & F501N & 3586 & & & \\
\hline \multirow[t]{4}{*}{2537} & Feb. 031994 & $\mathrm{PC} 2$ & Imaging & F255W & 2400 & 5203 & J. Trauger & Burrows et al. (1995) \\
\hline & & & & F502N & 2400 & & & \\
\hline & & & & F547M & 2400 & & & \\
\hline & & & & F656N & 2400 & & & \\
\hline \multirow[t]{8}{*}{2770} & Sep. 241994 & $\mathrm{PC} 2$ & Imaging & F255W & 1800 & 5753 & R. Kirshner & Panagia et al. (1996) \\
\hline & & & & F336W & 1200 & & & \\
\hline & & & & F439W & 800 & & & \\
\hline & & & & F502N & 4800 & & & \\
\hline & & & & F555W & 600 & & & \\
\hline & & & & F658N & 4800 & & & \\
\hline & & & & F675W & 600 & & & \\
\hline & & & & F814W & 600 & & & \\
\hline \multirow[t]{4}{*}{2875} & Jan. 071995 & FOS & Spectroscopy & $\mathrm{G} 190 \mathrm{H}$ & 5610 & 5753 & R. Kirshner & Chugai et al. (1997) \\
\hline & & & & $\mathrm{G} 270 \mathrm{H}$ & 6000 & & & \\
\hline & & & & $\mathrm{G} 400 \mathrm{H}$ & 4000 & & & \\
\hline & & & & $\mathrm{G} 570 \mathrm{H}$ & 4000 & & & \\
\hline \multirow[t]{4}{*}{2932} & Mar. 051995 & $\mathrm{PC} 2$ & Imaging & F439W & 800 & 5753 & R. Kirshner & \\
\hline & & & & F555W & 600 & & & \\
\hline & & & & F675W & 600 & & & \\
\hline & & & & F814W & 600 & & & \\
\hline 3043 & Jun. 241995 & FOC & Spectroscopy & NUV-prism & 16200 & 6130 & P. Jakobsen & (this paper) \\
\hline \multirow[t]{8}{*}{3270} & Feb. 061996 & $\mathrm{PC} 2$ & Imaging & $\mathrm{F} 255 \mathrm{~W}$ & 2300 & 6020 & R. Kirshner & \\
\hline & & & & F336W & 1200 & & & \\
\hline & & & & F439W & 950 & & & \\
\hline & & & & $\mathrm{F} 502 \mathrm{~N}$ & 7800 & & & \\
\hline & & & & F555W & 600 & & & \\
\hline & & & & F658N & 5200 & & & \\
\hline & & & & F675W & 600 & & & \\
\hline & & & & F814W & 600 & & & \\
\hline 3693 & Apr. 041997 & STIS & Imaging & OII & 2415 & 7122 & G. Sonneborn & \\
\hline \multirow[t]{7}{*}{3790} & Jul. 101997 & $\mathrm{PC} 2$ & Imaging & F255W & 2600 & 6437 & R. Kirshner & Garnavich et al. (1997a) \\
\hline & & & & F336W & 1600 & & & \\
\hline & & & & F439W & 800 & & & \\
\hline & & & & F555W & 600 & & & \\
\hline & & & & F656N & 5600 & & & \\
\hline & & & & F675W & 600 & & & \\
\hline & & & & $\mathrm{F} 814 \mathrm{~W}$ & 800 & & & \\
\hline 3792 & Jul. 121997 & & & F502N & 8200 & & & \\
\hline 3878 & Oct. 061997 & STIS & Spectroscopy & G430L & 5095 & 7434 & R. Kirshner & Garnavich et al. (1997b) \\
\hline 3879 & Oct. 071997 & & & G750M & 4772 & & & \\
\hline 3880 & Oct. 081997 & & & G230L & 14082 & & & \\
\hline \multirow[t]{7}{*}{4336} & Jan. 071999 & $\mathrm{PC} 2$ & Imaging & F255W & 7200 & 7434 & R. Kirshner & Garnavich et al. (1999) \\
\hline & & & & F336W & 2400 & & & \\
\hline & & & & F439W & 1000 & & & \\
\hline & & & & F555W & 600 & & & \\
\hline & & & & F656N & 7200 & & & \\
\hline & & & & F675W & 1200 & & & \\
\hline & & & & F814W & 800 & & & \\
\hline
\end{tabular}

Notes: ${ }^{\dagger}$ Since the SN outburst on Feb. 23, 1987; ${ }^{\ddagger}$ Total exposure time in seconds; *ID number of the observing program. 


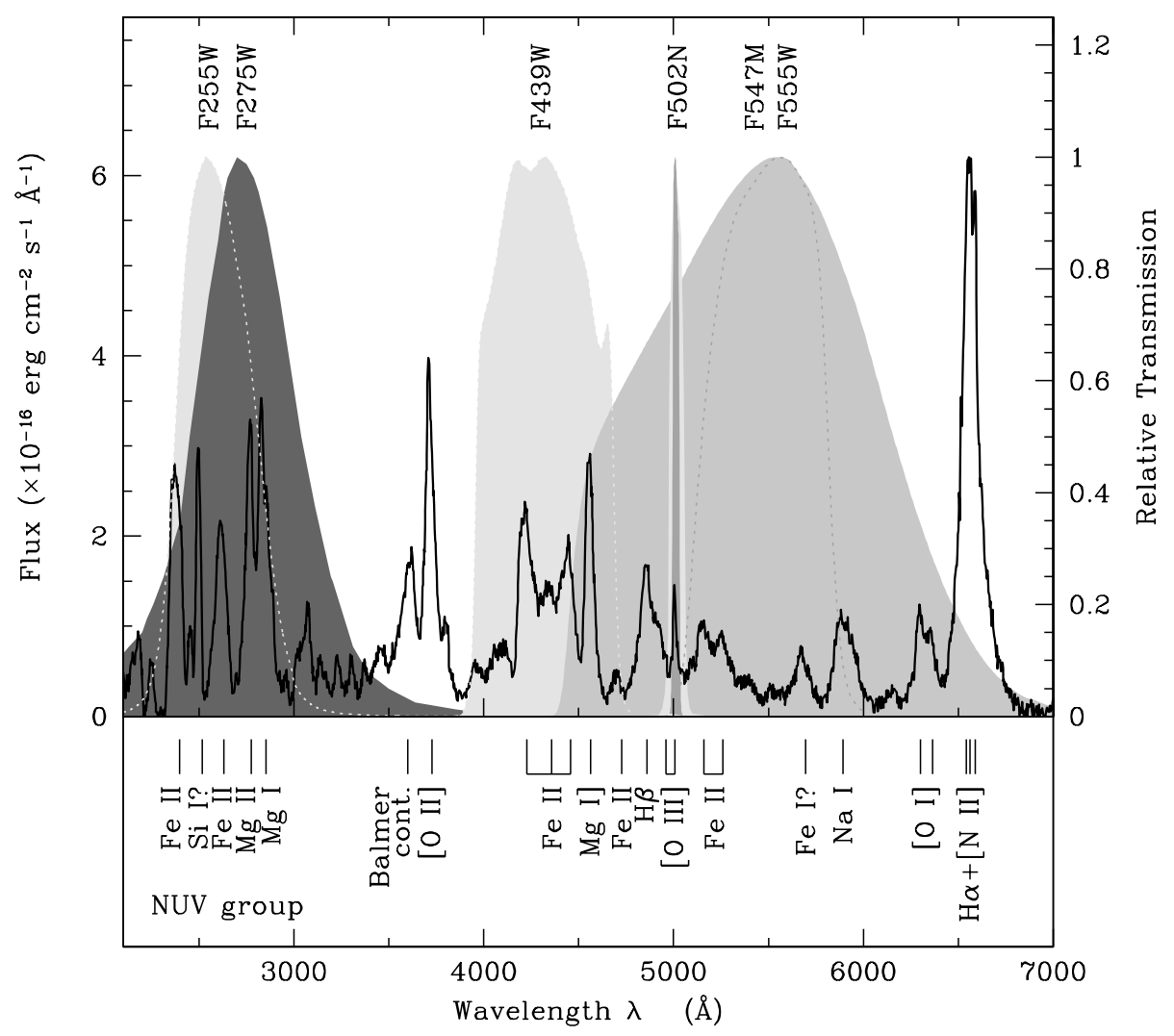

Fig. 3. Match of selected NUV and visible $H S T$ filters to spectral features in the SN 1987A spectrum. The spectrum shown is the day 2875 FOS spectrum presented by Chugai et al. (1997). The line identifications are from that paper and from Wang et al. (1996). The transmission axis (right) refers to the filters shown

\section{Results}

\subsection{Ellipticity of the $S N$ debris}

The FOC and PC2 imagery reveal that the shape of the SN debris in the visible became noticeably elongated after about day 2500. The later day $3792 \mathrm{~F} 502 \mathrm{~N}$ and the day $4336 \mathrm{~F} 555 \mathrm{~W}$ data show apparent axis ratios close to 0.7 , whereas the earliest day 1278 and 1754 FOC data were consistent with negligible elongation (Jakobsen et al. 1993). We limit ourselves in the present discussion to the large scale structure of the SN debris. To first order the shape of the debris can be described as elliptical. It is obvious from the best $\mathrm{S} / \mathrm{N}$ images, especially in the narrow-band filters, that there is fine-structure in the debris. Pun \& Kirshner (1996) tentatively described the debris shape as a dumbell-shaped structure. Accurate description of these features and monitoring of their evolution over time, however, would have required excessively long exposure times to obtain the necessary signal.

We derived ellipticities from the 2nd order image moments measured in a small image section centered on the SN remnant. If we denote the 2nd order image moments by $M_{x x}, M_{y y}$ and $M_{x y}$ then the ellipticity is given by:

$$
\epsilon=1-b / a=\frac{\sqrt{\left(M_{x x}-M_{y y}\right)^{2}+\left(2 M_{x y}\right)^{2}}}{\left(M_{x x}+M_{y y}\right)} .
$$

Initial measurements of the FWHM of the debris (see also Sect. 3.2) and the error thereon were used to define the minimum and maximum size of the image region in which to measure the ellipticities. The ellipticity was measured in a set of nested image sections with halfsizes that grow between FWHM- $\sigma_{\text {FWHM }}$ and FWHM $+\sigma_{\text {FWHM }}$ with increments of 1 pixel. The adopted ellipticities used in the analysis below are the weighted average of the individual measurements. These tend to be conservative in the sense that in the smaller image sections the measured ellipticity tends to be systematically higher than in the larger sections.

Figure 4a shows that the ellipticity in the [O III] narrow-band filters (FOC/F501N and PC2/F502N) increases approximately linearly with time. A weighted linear least-squares fit gives a rate of change $\mathrm{d} \epsilon / \mathrm{d} t$ of $(7.8 \pm 1.9) 10^{-5}$ day $^{-1}$ and onset of the elongation at day $(600 \pm 400)$. The large errors on the PC2 F502N measurements are due in part to the larger plate scale of the PC2 CCD (compared to the FOC camera), and in part to the low signal levels in the SN debris, as these data were obtained primarily for the purpose of studying the inner circumstellar ring.

The debris shape is dramatically less elongated in the near-UV (FOC/F275W and PC2/F255W) filters compared to the [O III] filter (Fig. 4b). It is possible that in the near-UV the ellipticity increases slowly with time, but low 

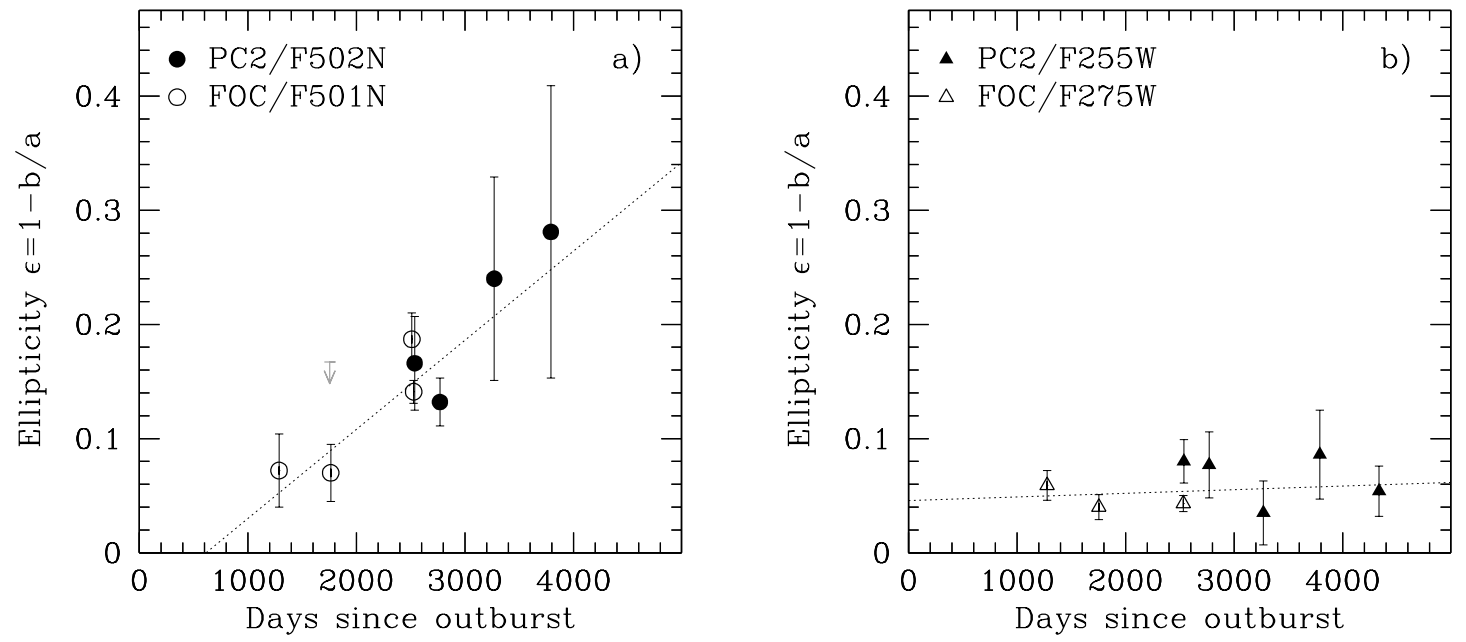

Fig. 4. Time evolution of the ellipticity $\epsilon=1-b / a$ of the debris of SN 1987A as a function of wavelength. a) Measurements of $\epsilon$ in the F501N (FOC) and F502N (PC2) filters with $\pm 1 \sigma$ error bars. The formal upper limit from Jakobsen et al. (1993) for the day $1754 \mathrm{~F} 501 \mathrm{~N}$ data is indicated by the grey arrow symbol. A weighted least-squares fit to the combined F501N and F502N data is overlayed. b) As a) for the F275W (FOC) and F255W (PC2) filters

surface brightness, low-signal, exposures preclude a definite measure. A small constant ellipticity $\epsilon=1-b / a \sim$ $0.05_{-0.02}^{+0.03}$ is consistent with the available data.

\subsection{Expansion of the $S N$ debris}

In order to quantify the expansion of the envelope of the SN debris in a consistent manner, we have to take its systematic change in elongation at the later stages into account. Also, considering the dimming and hence lower $\mathrm{S} / \mathrm{N}$ ratios of the more recent data, it is necessary to characterize the size by as robust an estimator as possible. We adopt the FWHM of the intensity profile of the debris, but calculated in terms of an elliptical or equivalent radius. If the semi-major and semi-minor axes at a given surface brightness level are denoted by $a$ and $b$, respectively, then the elliptical radius $r$ corresponding to that isophote is given by $r=\sqrt{a b}$. Given an ellipticity $\epsilon=1-b / a$ the elliptical radius is related to the semi-major axis radius by $r=a \sqrt{1-\epsilon}$.

For each epoch of observation and each filter, we performed aperture photometry in a set of nested circular apertures and constructed radial intensity profiles by differencing the signal in consecutive apertures. The FWHM measured in these radial intensity profiles was expressed in terms of elliptical radii by multiplication with the geometric correction term $\sqrt{1-\epsilon_{\lambda}(t)}$. For $\epsilon_{\lambda}(t)$ we adopted the empirical least-squares fits shown for F501N/F502N and F255W/F275W filters in Figs. 4, and similar fits for the other available filters. Using a simple geometric correction was possible because the spatial extent of the debris is much larger than the PSF, and the distribution of the light is fairly smooth and not very concentrated.

In Figs. 5a and b we present the resulting measures of the size of the SN debris as a function of time for both the visible (F501N, F502N, F547M and F555W) and near-
UV (F255W and F275W) data sets. Also plotted are the equivalent "two dimensional" spectroscopic measures derived from the day 3043 FOC near-UV prism data shown in Fig. 2, and a high-quality near-UV point derived in the same way from the day 3880 STIS G230L-grating spectrum. In the case of the spectroscopic measures we also applied a (small) geometric correction to account for the mismatch of the position angles of the dispersion axis and the major axis of the $\mathrm{SN}$ debris.

The best-fit linear expansion rates are $(10.34 \pm 0.84)$ mas yr$^{-1}$ for the combined visible, and $(16.27 \pm 0.83)$ mas $y r^{-1}$ for the combined near-UV data. For a distance to SN 1987A of $51 \mathrm{kpc}$ (Panagia et al. 1991) this corresponds to effective expansion velocities of $\sim 2500$ and $\sim 3930 \mathrm{~km} \mathrm{~s}^{-1}$, respectively. These values match the earlier results obtained by Jakobsen et al. (1994) from the FOC data of days 1278, 1754 and 2522 to within the respective errors.

\subsubsection{Deviations from linear expansion?}

The FOC objective prism observations presented above leave little doubt that the larger apparent size of the SN 1987A envelope in the near-UV is due to the emission at these wavelengths being subjected to multiple scattering and reprocessing in the optically thick resonance transitions of $\mathrm{MgI} / \mathrm{Mg} \mathrm{II} / \mathrm{Fe}$ II ions located in the outer, faster moving regions of the ejecta as suggested by Wang et al. (1996) and Chugai et al. (1997).

However, as pointed out by the latter authors, in this case one does not expect the outermost regions of the ejecta to grow in a strictly linear fashion. Specifically, in the case of conservative scattering in a linearly expanding envelope displaying a power-law density profile $\rho(r) \propto r^{-k}$, the radius at which optical depth unity is reached is expected to increase with time as $r(t) \propto t^{\frac{k-3}{k-1}}$. 

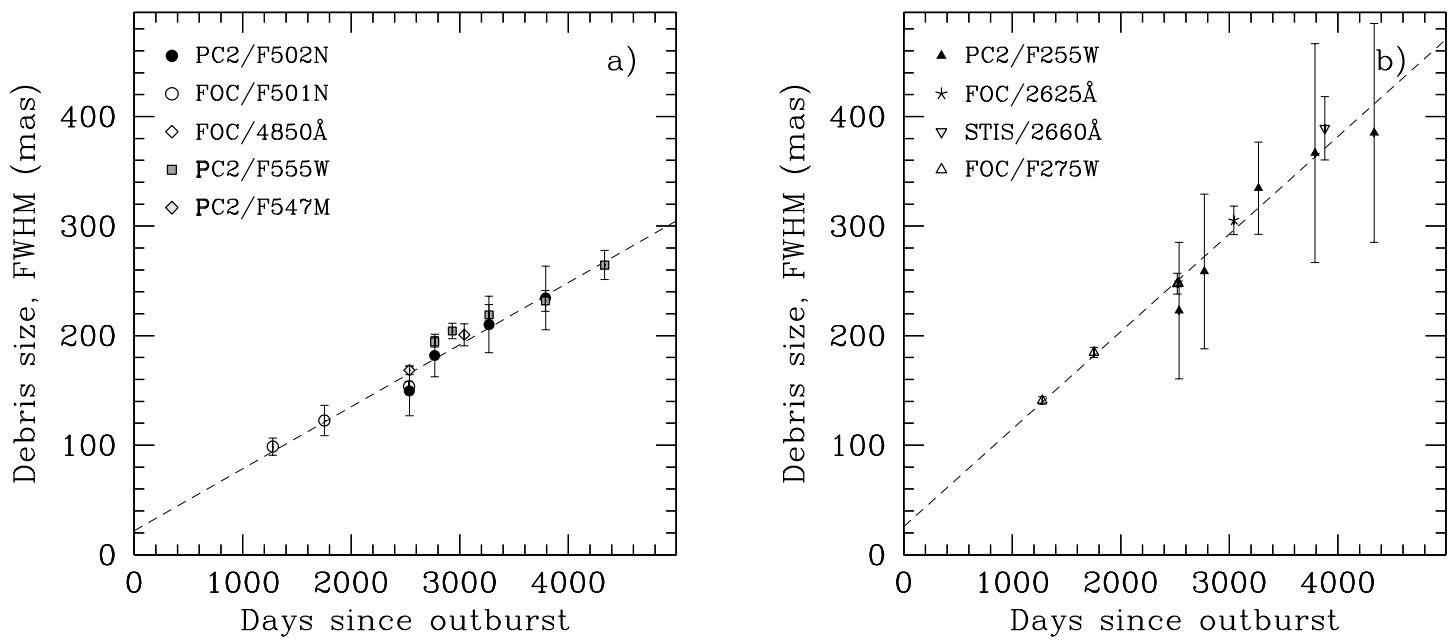

Fig. 5. The apparent expansion of the ejecta of SN 1987A, as characterized by the increase of the elliptical FWHM diameter (see text) of the envelope with time. a) The F501N (FOC) and F502N (PC2) filters. Also shown are measurements for the F547M and F555W (PC2) passbands and measurements for the spectroscopic equivalent in the day 3034 FOC data (central wavelength $\left.\lambda_{c}=4850 \AA\right)$. Error bars denote $\pm 1 \sigma$. The dashed line indicates the best-fit linear expansion rate. b) as a) for the F275W (FOC) and F255W (PC2) filters. Also shown are a FOC $\left(\lambda_{c}=2625 \AA\right)$ and a STIS $\left(\lambda_{\mathrm{c}}=2660 \AA\right)$ data point for the spectroscopic equivalent of these filters. A linear fit (dashed) is overlayed.

As discussed by Chugai et al. (1997), optical depth unity in the $\mathrm{Mg}$ II line is reached in the very outmost regions of the ejecta corresponding to expansion velocities of order $\sim 9000 \mathrm{~km} \mathrm{~s}^{-1}$, where most models assume a very steep density gradient corresponding to $k \simeq 9$. Hence Chugai et al. predicted that outer boundary of the envelope of SN 1987A should expand more slowly than linearly; i.e., as $r(t) \propto t^{0.75}$ in the near-UV.

As is evident from Fig. 5, the growth of the size of the SN 1987A debris - when defined in terms of the FWHM of the images - remains consistent with linear expansion out to the last available data point (day 4336; 1999 Jan. 7) in both the visible and the near-UV. However, these measures probe velocities in the range $\sim 2500-4000 \mathrm{~km} \mathrm{~s}^{-1}$. To see the effect predicted by Chugai et al. we need to go 2-3 times further out in the images, corresponding to angular radii of order $\sim 300$ mas and brightness levels $\sim 10 \%$ of peak and fainter.

For $k \simeq 9$ the anticipated deviation from linearity at these large radii is only of order $\sim 11 \%$ between day $\sim 2500$ (and the last high-quality FOC F275W imaging data) and day $\sim 4000$ (and the last PC2 F255W images). Unfortunately, the available late-time HST data on SN 1987A do not allow us to detect such a relatively subtle effect. As is clear from Fig. 2, the outer regions of interest beyond $\sim 200$ mas radius in the FOC prism data are seriously hampered by contamination from the dispersed images of star 3 and the circumstellar ring. Likewise, as is evident from Fig. $5 \mathrm{~b}$ the $\mathrm{S} / \mathrm{N}$ ratio of the later day $\mathrm{PC} 2$ and STIS data are too low to permit a measure of the image sizes at, say, $10 \%$ peak at the required accuracy.

We conclude that there is unfortunately no chance of detecting the effect predicted by Chugai et al. in the latetime $H S T$ data - and that the apparent linear expansion displayed in Fig. 5 therefore does not challenge the res- onance scattering explanation for the wavelength dependence of the size of the SN 1987A debris.

\section{Conclusions}

We have presented HST FOC near-UV objective prism observations of the ejecta of SN 1987A taken on 1995 June 24 (3043 days after the SN outburst). We have combined these data with available archival FOC, WFPC2, and STIS data to study the late-time expansion of the SN debris over a period of $\sim 8$ years. Our main findings are:

1) Provided the pronounced ellipticity of the SN image seen in the visible data is taken into account, the available data are consistent with the SN envelope having expanded linearly in time at all wavelengths out to the last data point sampled (1999 January 7; day 4336).

2) Throughout this expansion, the apparent size of the ejecta, expressed in terms of the FWHM of the radial intensity profile, remained some $\sim 50 \%$ larger in the near-UV than in the visible.

3) The FOC near-UV prism spectrum reveals that the large spatial extent of the SN 1987A image is confined to the 2350-2900 $\AA$ wavelength region containing the resonance lines of $\mathrm{Mg}$ I, $\mathrm{Mg}$ II and Fe II, thereby confirming the suggestion of Wang et al. (1996) and Chugai et al. (1997) that the larger apparent size of the SN 1987A envelope in the near-UV is due to multiple scattering and reprocessing in these transitions in the outer regions of the envelope.

\section{References}

Burrows, C. J., et al. 1995, ApJ, 452, 680

Chugai, N. N., Chevalier, R. A., Kirshner, R. P., \& Challis, P. 1997, ApJ, 483, 925

Crotts, A. P. S., Kunkel, W. E., \& Heathcote, S. R. 1995, ApJ, 438,724 
Crotts, A. P. S., \& Heathcote, S. R. 2000, ApJ, 528, 426

Garnavich, P., Kirshner, P., Challis, P., et al. 1997a, IAU Circ., 6710, 2

Garnavich, P., Kirshner, P., Challis, P., et al. 1997b, IAU Circ., 6761,1

Garnavich, P., Kirshner, P., Challis, P., et al. 1999, IAU Circ., 7102, 1

Jakobsen, P., Albrecht, R., Barbieri, C., et al. 1991, ApJ, 369, L63

Jakobsen, P., Macchetto, F., \& Panagia, N. 1993, ApJ, 403, 736

Jakobsen, P., Jedrzejewski, R., Macchetto, F., \& Panagia, N. 1994, ApJ, 435, L47

Luo, D., McCray, R., \& Slavin, J. 1994, ApJ, 430, 264

Michael, E., McCray, R., Pun, C. S. J., et al. 2000, ApJ, 542, L53
Panagia, N., Gilmozzi, R., Macchetto, F., Adorf, H. M., \& Kirshner, R. P. 1991, ApJ, 380, L23

Panagia, N., Scuderi, S., Gilmozzi, R., et al. 1996, ApJ, 459, L17

Plait, P., Chevalier, R., \& Kirshner, R. P. 1992, IAU Circ., No. 5592

Plait, P. C., Lundqvist, P., Chevalier, R. A., \& Kirshner, R. P. 1995, ApJ, 439, 730

Pun, C. S. J., \& Kirshner, R. P. 1996, AAS, 189, 4504

Sonneborn, G., Pun, C. S. J., Kimble, R. A., et al. 1998, ApJ, 492, L139

Wang, L., Wheeler, J. G., Kirshner, R. P., et al. 1996, ApJ, 466, 998

West, R. M., Lauberts, A., Jørgensen, H. E., \& Schuster, H.-E. 1987, A\&A, 177, L1 